\title{
Entropy growth of shift-invariant states on a quantum spin chain
}

\author{
M. Fannes ${ }^{1, *}$, B. Haegeman ${ }^{1, \dagger}$, M. Mosonyi ${ }^{2, \ddagger}$ \\ October 31, 2018 \\ ${ }^{1}$ Instituut voor Theoretische Fysica \\ K.U.Leuven \\ Celestijnenlaan 200D, B-3001 Heverlee, Belgium \\ ${ }^{2}$ Mathematical Institute \\ Budapest University of Technology and Economics \\ H-1521 Budapest XI. Sztoczek u. 2, Hungary
}

\begin{abstract}
We study the entropy of pure shift-invariant states on a quantum spin chain. Unlike the classical case, the local restrictions to intervals of length $N$ are typically mixed and have therefore a non-zero entropy $S_{N}$ which is, moreover, monotonically increasing in $N$. We are interested in the asymptotics of the total entropy. We investigate in detail a class of states derived from quasi-free states on a CAR algebra. These are characterised by a measurable subset of the unit interval. As the entropy density is known to vanishes, $S_{N}$ is sublinear in $N$. For states corresponding to unions of finitely many intervals, $S_{N}$ is shown to grow slower than $(\log N)^{2}$. Numerical calculations suggest a $\log N$ behaviour. For the case with infinitely many intervals, we present a class of states for which the entropy $S_{N}$ increases as $N^{\alpha}$ where $\alpha$ can take any value in $(0,1)$.
\end{abstract}

\section{Introduction}

In quantum statistical mechanics, one-dimensional lattice systems, the so-called spin chains, are far from fully understood. One of the obstacles for a systematic study

\footnotetext{
*E-mail: mark.fannes@fys.kuleuven.ac.be

${ }^{\dagger}$ E-mail: bart.haegeman@fys.kuleuven.ac.be

‡E-mail: mosonyi@chardonnay.math.bme.hu
} 
is the complicated correlations that can occur. This is even possible for pure states, which are trivial for classical spin chains. Due to these quantum correlations, it is often very hard to explicitly specify a state. Only a few classes can be studied in detail, including the product states, the finitely correlated states [4] and the states derived from quasi-free states on the CAR algebra [2, 3].

Let us denote by $\rho_{N}$ the density matrix of the restriction of a translation-invariant state $\rho$ on a spin chain to $N$ consecutive spins. The von Neumann entropy $S_{N}:=$ $S\left(\rho_{N}\right)$ has proved to be a very useful quantity in the study of quantum correlations. For ergodic translation-invariant states, $\rho_{N}$ is essentially concentrated on a subspace of dimension $\exp (N s(\rho))$ [6]. Here, $s(\rho)$ is the entropy density of $\rho$. The compression of $\rho_{N}$ from the full dimension $d^{N}$ of $N$ spins to $\exp (N s(\rho))$ lies e.g. at the basis of DMRG computations [8]. One may conjecture that $s(\rho)=0$ for pure states $\rho$, which should allow for a very efficient compression. For pure states, $S_{N}$ is also the unique reasonable measure for the entanglement of this interval with the rest of the chain [7] and it measures therefore the resources of the state for quantum computing purposes.

For pure product states $S_{N}$ vanishes for all $N$, this is in fact completely analogous to the classical spin chain. For pure finitely correlated states $S_{N}$ is uniformly bounded, a behaviour that is certainly not expected to be generic.

In this paper, we study the entropy $S_{N}$ for translation-invariant pure states derived from quasi-free states on the CAR algebra. Here, the entropy density is known to vanish and we investigate the sublinear growth of the entropy $S_{N}$ when $N \rightarrow \infty$. We show that $S_{N}$ increases much faster with $N$ than in the previous cases. For the simplest quasi-free states, the entropy behaves as $\log N$. We shall also present a more involved example for which the entropy increases as $N^{\alpha}$ with $\alpha$ arbitrarily close to 1 .

The construction of pure shift-invariant quasi-free states is recalled in Section 2 Such states are characterised by a subset of the unit interval. In Section 3 we prove that the asymptotics of $S_{N}$ as $N \rightarrow \infty$ can be obtained by a quadratic approximation of the entropy. The entropy growth of quasi-free states given by a set consisting of finitely many intervals is studied in Section 4. Finally, Section 5 is devoted to the infinitely many intervals case.

\section{Quasi-free states on the spin chain}

In this section we show, following [3], how a quasi-free state on the CAR algebra can be used to define a state on the spin chain algebra. After the introductory definitions, we explain how both algebras can be retrieved as subalgebras of a larger algebra. This construction permits to transfer translation-invariant states from the CAR algebra to the spin chain algebra. This idea is then applied to quasi-free states. 


\subsection{CAR algebra and spin chain algebra}

Let $\mathcal{H}$ be the Hilbert space $\ell^{2}(\mathbb{Z})$, in which $\left\{\delta_{k}: k \in \mathbb{Z}\right\}$ forms an orthonormal basis, where $\delta_{k}$ is the characteristic function of the integer number $k$. Let $\mathcal{A}$ be the CAR algebra corresponding to $\mathcal{H}$. It is the $\mathrm{C}^{*}$-algebra generated by $\mathbb{1}$ and $\left\{c_{k}: k \in\right.$ $\mathbb{Z}\}$, satisfying the canonical anticommutation relations

$$
c_{k} c_{l}=-c_{l} c_{k} \quad c_{k}^{*} c_{l}=\delta_{k, l} \mathbb{1}-c_{l} c_{k}^{*}
$$

The parity automorphism $\alpha$ on $\mathcal{A}$ is defined by $\alpha\left(c_{k}\right)=-c_{k}$. Let $\mathcal{A}_{+}$be the fixed point algebra of $\alpha$, i.e. $\mathcal{A}_{+}=\{a \in \mathcal{A}: \alpha(a)=a\}$. The elements of $\mathcal{A}_{+}$are called even, while those of $\mathcal{A}_{-}:=\{a \in \mathcal{A}: \alpha(a)=-a\}$ are odd. Obviously, $\mathcal{A}=\mathcal{A}_{+}+\mathcal{A}_{-}$. The shift automorphism $\gamma$ is defined by $\gamma\left(c_{k}\right)=c_{k+1}$.

The quantum spin chain is the UHF algebra

$$
\mathcal{C}:=\bigotimes_{k=-\infty}^{+\infty} \mathcal{M}_{2}
$$

where $\mathcal{M}_{2}$ is the algebra of $2 \times 2$ matrices. Let $e_{11}^{k}, e_{12}^{k}, e_{21}^{k}$ and $e_{22}^{k}$ denote the standard matrix units of $\mathcal{M}_{2}$ embedded into the $k$ th factor of $\mathcal{C}$. The following relations hold:

$$
\begin{aligned}
e_{a b}^{k} e_{c d}^{l} & =e_{c d}^{l} e_{a b}^{k} \quad \text { when } k \neq l, \\
e_{a b}^{k} e_{c d}^{k} & =\delta_{b, c} e_{a d}^{k}, \\
\left(e_{a b}^{k}\right)^{*} & =e_{b a}^{k} \\
e_{11}^{k}+e_{22}^{k} & =\mathbb{1} .
\end{aligned}
$$

Any algebra generated by elements $\left\{E_{a b}^{k}: a, b \in\{1,2\}, k \in \mathbb{Z}\right\}$ satisfying the above relations is isomorphic to $\mathcal{C}$.

\subsection{Jordan-Wigner isomorphism}

Let $\mathcal{A}_{n}$ be the algebra generated by $\left\{c_{k}: 0 \leq k \leq n-1\right\}$ and let $\mathcal{C}_{n}=\bigotimes_{k=0}^{n-1} \mathcal{M}_{2}$. It is well-known that $\mathcal{A}_{n}$ is isomorphic to $\mathcal{C}_{n}$ for all $n \in \mathbb{N}$. An explicit isomorphism is given by the so-called Jordan-Wigner isomorphism given in terms of matrix units in $\mathcal{A}_{n}$ by

$$
E_{11}^{k}:=c_{k}^{*} c_{k}, \quad E_{22}^{k}:=c_{k} c_{k}^{*}, \quad E_{12}^{k}:=A_{k} c_{k}^{*}, \quad E_{21}^{k}:=A_{k} c_{k} .
$$

Here we introduced

$$
\sigma_{k}^{z}:=2 c_{k}^{*} c_{k}-\mathbb{1}, \quad A_{k}:=\prod_{l=0}^{k-1} \sigma_{l}^{z} .
$$


The set $\left\{E_{a b}^{k}: a, b \in\{1,2\}, 0 \leq k \leq n-1\right\}$ generates $\mathcal{A}_{n}$ and the operators $E_{a b}^{k}$ satisfy the same relations (1D) as the matrix units $e_{a b}^{k}$ of $C_{n}$.

A first idea would be to extend this isomorphism to an isomorphism from $\mathcal{A}$ to $\mathcal{C}$. However, it is impossible to extend this definition to negative $k$ 's in such a way that the isomorphism intertwines the shifts in $\mathcal{A}$ and $\mathcal{C}$. This property is needed to transport translation-invariance from $\mathcal{A}$ to $\mathcal{C}$.

One way to circumvent this problem is the following. Enlarge $\mathcal{A}$ to $\hat{\mathcal{A}}$ by adding a new element $T$ that has the following properties

$$
\begin{aligned}
& T^{*}=T, \quad T^{2}=\mathbb{1} \quad \text { (i.e. } T \text { is a self-adjoint unitary) } \\
& T c_{k} T= \begin{cases}c_{k} & \text { if } k \geq 0 \\
-c_{k} & \text { if } k<0 .\end{cases}
\end{aligned}
$$

Any element of $\hat{\mathcal{A}}$ can uniquely be written in the form $a+T b$ with $a$ and $b$ from $\mathcal{A}$. Therefore, $\hat{\mathcal{A}}=\mathcal{A}+T \mathcal{A}$. Note that formally $T=\prod_{k=-1}^{-\infty} \sigma_{k}^{z}$.

A state $\varphi$ on $\mathcal{A}$ can be extended to a state $\hat{\varphi}$ on $\hat{\mathcal{A}}$ by $\hat{\varphi}(a+T b):=\varphi(a)$ and the extensions of the automorphisms $\alpha$ and $\gamma$ are

$$
\hat{\alpha}(a+T b):=\alpha(a)+T \alpha(b) \quad \text { and } \quad \hat{\gamma}(a+T b):=\gamma(a)+T \sigma_{0}^{z} \gamma(b) .
$$

We define another automorphism $\beta$ on $\hat{\mathcal{A}}$ by $\beta(a+T b):=a-T b$. The fixed point algebra of $\beta^{-1} \hat{\alpha}$ will be denoted by $\check{\mathcal{A}}$, i.e.,

$$
\begin{aligned}
\check{\mathcal{A}} & =\{a+T b \in \hat{\mathcal{A}}: \hat{\alpha}(a+T b)=\beta(a+T b)\} \\
& =\{a+T b \in \hat{\mathcal{A}}: \alpha(a)=a, \alpha(b)=-b\} \\
& =\mathcal{A}_{+}+T \mathcal{A}_{-} .
\end{aligned}
$$

The restriction of a state $\hat{\varphi}$ on $\hat{\mathcal{A}}$ to a state on $\breve{\mathcal{A}}$ will be denoted by $\check{\varphi}$. Because the automorphisms $\hat{\alpha}$ and $\hat{\gamma}$ leave the subalgebra $\check{\mathcal{A}}$ invariant, they can be restricted to $\check{\mathcal{A}}$. Denote these restrictions by $\check{\alpha}$ and $\check{\gamma}$.

Let $\varphi$ be an even state, i.e., it vanishes on odd elements or, equivalently, $\varphi \circ \alpha=\varphi$. It is easy to see that also $\check{\varphi} \circ \check{\alpha}=\check{\varphi}$, thus $\check{\varphi}$ is an even state on $\check{\mathcal{A}}$. Similarly, let $\varphi$ be a translation-invariant state on $\mathcal{A}$, i.e. $\varphi \circ \gamma=\varphi$, then $\check{\varphi} \circ \check{\gamma}=\check{\varphi}$, thus $\check{\varphi}$ is a translation-invariant state on $\check{\mathcal{A}}$.

Now, define

$$
\tilde{E}_{11}^{k}:=c_{k}^{*} c_{k}, \quad \tilde{E}_{22}^{k}:=c_{k} c_{k}^{*}, \quad \tilde{E}_{12}^{k}:=T A_{k} c_{k}^{*}, \quad \tilde{E}_{21}^{k}:=T A_{k} c_{k} .
$$

with

$$
\sigma_{k}^{z}:=2 c_{k}^{*} c_{k}-\mathbb{1}, \quad A_{k}:= \begin{cases}\prod_{\ell=0}^{k-1} \sigma_{\ell}^{z} & \text { if } k>0 \\ \mathbb{1} & \text { if } k=0 \\ \prod_{\ell=k}^{-1} \sigma_{\ell}^{z} & \text { if } k<0\end{cases}
$$


One verifies that these operators satisfy the same commutation relations as the matrix units of $\mathcal{C}$. Moreover, $\check{\gamma}\left(\tilde{E}_{a b}^{k}\right)=\tilde{E}_{a b}^{k+1}$.

To summarise, we constructed an algebra $\hat{\mathcal{A}}$ which contains both $\mathcal{A}$ and $\check{\mathcal{A}}$ as subalgebras. This embedding is compatible with the translations on the subalgebras. Moreover, we established an isomorphism between $\breve{\mathcal{A}}$ and $\mathcal{C}$ which is also compatible with the translations. This allows us to transfer translation-invariant states from $\mathcal{A}$ to $\mathcal{C}$.

Let $\varphi$ be a translation-invariant state. Such a state is automatically even and is completely determined by the sequence $\left(\varphi_{n}\right)_{n=0}^{+\infty}$, where $\varphi_{n}$ is the restriction of $\varphi$ to $\mathcal{A}_{n}$. The density matrix $\left[\varphi_{n}\right]$ of $\varphi_{n}$ has entries

$$
\left[\varphi_{n}\right]_{\underline{j}, \underline{i}}=\varphi\left(\prod_{k=0}^{n-1} E_{i_{k} j_{k}}^{k}\right), \quad \underline{i}, \underline{j} \in\{1,2\}^{n} .
$$

The transferred state $\check{\varphi}$ is also translation-invariant and so completely determined by its restriction to the subalgebras $\left\{\mathcal{C}_{n}: n \in \mathbb{N}\right\}$ with density matrices

$$
\left[\check{\varphi}_{n}\right]_{\underline{j}, \underline{i}}=\hat{\varphi}\left(\prod_{k=0}^{n-1} \tilde{E}_{i_{k} j_{k}}^{k}\right) \quad \underline{i}, \underline{j} \in\{1,2\}^{n} .
$$

The expressions $\prod_{k=0}^{n-1} E_{i_{k} j_{k}}^{k}$ and $\prod_{k=0}^{n-1} \tilde{E}_{i_{k} j_{k}}^{k}$ are both either odd or even. When odd, $\left[\varphi_{n}\right]_{\underline{j, \underline{i}}}=\left[\check{\varphi}_{n}\right]_{j, \underline{i}}=0$, while when even, since $T c_{k}=c_{k} T$ for $k \geq 0$ and $T^{2}=\mathbb{1}$, we get that $\prod_{k=0}^{n-1} \tilde{E}_{i_{k} j_{k}}^{\bar{k}}=\prod_{k=0}^{n-1} E_{i_{k} j_{k}}^{k}$ and so $\left[\varphi_{n}\right]_{\underline{i}, \underline{j}}=\left[\check{\varphi}_{n}\right]_{\underline{i}, \underline{j}}$. From this, we conclude that the states $\varphi$ and $\check{\varphi}$ have the same reduced density matrices. It follows immediately that if $\varphi$ is pure, then also $\check{\varphi}$ is pure.

\subsection{Quasi-free states}

We apply the construction of the previous section to quasi-free states on the CAR algebra $\mathcal{A}$. For these states an explicit formula is known for the entropy of the restricted density matrices. Because the corresponding states on $\mathcal{C}$ have the same restricted density matrices, the same explicit formulas are available, as we shall use in the following sections. The proofs of the theorems mentioned in this subsection can be found in [1].

Let $\varphi$ be a quasi-free, gauge-invariant state on $\mathcal{A}$, i.e., $\varphi$ is given by the rule

$$
\varphi\left(c_{i_{1}}^{*} \ldots c_{i_{m}}^{*} c_{j_{n}} \ldots c_{j_{1}}\right)=\delta_{m, n} \operatorname{det}\left(\left[Q_{i_{k} j_{l}}\right]_{k, l=1}^{n}\right)
$$

where $Q$ is an operator on $\mathcal{H}, 0 \leq Q \leq \mathbb{1}$ and $Q_{i j}=\left\langle\delta_{i}, Q \delta_{j}\right\rangle$ are the matrix elements of $Q$ in the standard basis of $\mathcal{H}$. The operator $Q$ is called the symbol of the state $\varphi$. Obviously, $\varphi$ is even. 
The quasi-free state $\varphi$ is translation-invariant if and only if its symbol $Q$ is a Toeplitz operator, i.e., there exists a sequence $\left\{q_{k}: k \in \mathbb{Z}\right\}$ such that $Q_{l k}=q(k-l)$. By the Fourier transform,

$$
q^{\wedge}(\theta)=\sum_{k \in \mathbb{Z}} q(k) \mathrm{e}^{i 2 \pi k \theta} \quad \text { and its inverse } \quad q(k)=\int_{\mathbb{T}} d \theta q^{\wedge}(\theta) \mathrm{e}^{-i 2 \pi k \theta},
$$

with $\mathbb{T}$ the torus parametrised by $[0,1)$, the symbol of a translation-invariant quasifree state is unitarily equivalent with the multiplication operator by $q^{\wedge}$ on $L^{2}(\mathbb{T}, d \theta)$. This function $q^{\wedge}$ satisfies $0 \leq q^{\wedge}(\theta) \leq 1$ almost everywhere.

A quasi-free state $\varphi$ is pure if and only if its symbol $Q$ is a projector. For a translationinvariant state this means that the Fourier transform of the symbol $Q$ is a characteristic function, i.e., there exists a measurable set $K \subset \mathbb{T}$ such that $q^{\wedge}(\theta)=\chi_{K}(\theta)$.

The entropy of a quasi-free state $\varphi$ can be expressed in terms of its symbol $Q$. Define, for $0 \leq x \leq 1$, the functions $\eta(x):=-x \log x$ and $\tilde{\eta}(x):=\eta(x)+\eta(1-x)$. The von Neumann entropy of the state restricted to an interval of $N$ spins is given by

$$
S_{N}:=\operatorname{Tr} \eta\left(\left[\varphi_{N}\right]\right)=\operatorname{Tr} \tilde{\eta}\left(Q_{N}\right)
$$

where $Q_{N}$ is the restriction of $Q$ to the $N$-dimensional space spanned by $\left\{\delta_{k}: 0 \leq\right.$ $k \leq N-1\}$. It follows by Szegö's theorem [ $[5]$ that the entropy density of a translationinvariant quasi-free state equals

$$
s:=\lim _{N \rightarrow \infty} \frac{S_{N}}{N}=\int d \theta \tilde{\eta}\left(q^{\wedge}(\theta)\right) .
$$

In particular, the entropy density of a pure translation-invariant quasi-free state is zero.

\section{Asymptotics for entropy of quasi-free states}

Quasi-free states are good approximations of true ground and equilibrium states for systems of Fermions, either at low density or with weakly interacting particles. The coordinate $\theta$ appearing in (21) has the meaning of momentum and the system is specified by a dispersion relation $\theta \mapsto \varepsilon(\theta)$ which is the relation between effective energy and momentum. For a shift-invariant quasi-free state, determined by a symbol $Q$ or, equivalently, by a measurable function $q^{\wedge}$ on the unit circle with $0 \leq q^{\wedge} \leq 1$, the energy and particle densities are given by

$$
e\left(\varepsilon, q^{\wedge}\right):=\int_{\mathbb{T}} d \theta \varepsilon(\theta) q^{\wedge}(\theta) \quad \text { and } \quad n\left(q^{\wedge}\right):=\int_{\mathbb{T}} d \theta q^{\wedge}(\theta) .
$$


The ground state at density $\lambda, 0 \leq \lambda \leq 1$, is obtained by minimising the energy density under the constraint $n\left(q^{\wedge}\right)=\lambda$. It is given by $q^{\wedge}=\chi_{K\left(e_{\mathrm{F}}(\lambda)\right)}$, where $K(e):=$ $\{\theta \in \mathbb{T}: \varepsilon(\theta) \leq e\}$ and $e_{\mathrm{F}}(\lambda)$ is the Fermi level determined by the condition

$$
\left|K\left(e_{\mathrm{F}}(\lambda)\right)\right|=\int_{\varepsilon(\theta) \leq e_{\mathrm{F}}(\lambda)} d \theta=\lambda
$$

For smooth dispersion relations with few oscillations in $\theta, K\left(e_{\mathrm{F}}(\lambda)\right)$ will typically consist of a finite union of disjoint intervals. This case will be investigated in Section 4 , Section 5 deals with the opposite situation when $K\left(e_{\mathrm{F}}(\lambda)\right)$ has a Cantor-like structure.

The quasi-free states on the spin chain $\mathcal{C}$, as introduced in the previous section, obey Equation (3) for the von Neumann entropy of the restricted density matrices. This will be the starting point for our study of the asymptotic behaviour of this entropy $S_{N}$ as $N \rightarrow \infty$.

\subsection{Growth exponents}

We use the following estimate for the entropy function $\tilde{\eta}(x):=\eta(x)+\eta(1-x)$,

$$
x(1-x) \leq \tilde{\eta}(x) \leq \epsilon-c \log \epsilon x(1-x), \quad 0 \leq x \leq 1,
$$

see Figure 1. The upper bound for $\tilde{\eta}$ holds for $c$ a constant independent of $0<\epsilon$, moreover, for $0<\epsilon<\epsilon_{0}$ we may choose $c=1+\mathrm{o}\left(\epsilon_{0}\right)$. Therefore,

$$
\operatorname{Tr} Q_{N}\left(\mathbb{1}-Q_{N}\right) \leq S_{N} \leq \epsilon N-c \log \epsilon \operatorname{Tr} Q_{N}\left(\mathbb{1}-Q_{N}\right) .
$$

By choosing a function $\epsilon(N)$ for which $\epsilon \rightarrow 0$ as $N \rightarrow \infty$, we obtain bounds for the entropy $S_{N}$ in terms of $\operatorname{Tr} Q_{N}\left(\mathbb{1}-Q_{N}\right)$. E.g., putting $\epsilon(N):=\frac{1}{N}$,

$$
\operatorname{Tr} Q_{N}\left(\mathbb{1}-Q_{N}\right) \leq S_{N} \leq 1+c \log N \operatorname{Tr} Q_{N}\left(\mathbb{1}-Q_{N}\right) .
$$

We are particularly interested in the growth exponents of the entropy,

$$
\alpha_{-}:=\liminf _{N \rightarrow+\infty} \frac{\log S_{N}}{\log N} \quad \text { and } \quad \alpha_{+}:=\limsup _{N \rightarrow+\infty} \frac{\log S_{N}}{\log N} .
$$

With the inequalities (4),

$$
\liminf _{N \rightarrow+\infty} \frac{\log \operatorname{Tr} Q_{N}\left(\mathbb{1}-Q_{N}\right)}{\log N} \leq \alpha_{-} \leq \alpha_{+} \leq \limsup _{N \rightarrow+\infty} \frac{\log \operatorname{Tr} Q_{N}\left(\mathbb{1}-Q_{N}\right)}{\log N} .
$$

We conclude that, if

$$
\begin{gathered}
\lim _{N \rightarrow+\infty} \frac{\log \operatorname{Tr} Q_{N}\left(\mathbb{1}-Q_{N}\right)}{\log N} \text { exists, then also } \lim _{N \rightarrow+\infty} \frac{\log S_{N}}{\log N} \quad \text { exists, and } \\
\alpha:=\lim _{N \rightarrow+\infty} \frac{\log S_{N}}{\log N}=\lim _{N \rightarrow+\infty} \frac{\log \operatorname{Tr} Q_{N}\left(\mathbb{1}-Q_{N}\right)}{\log N} .
\end{gathered}
$$




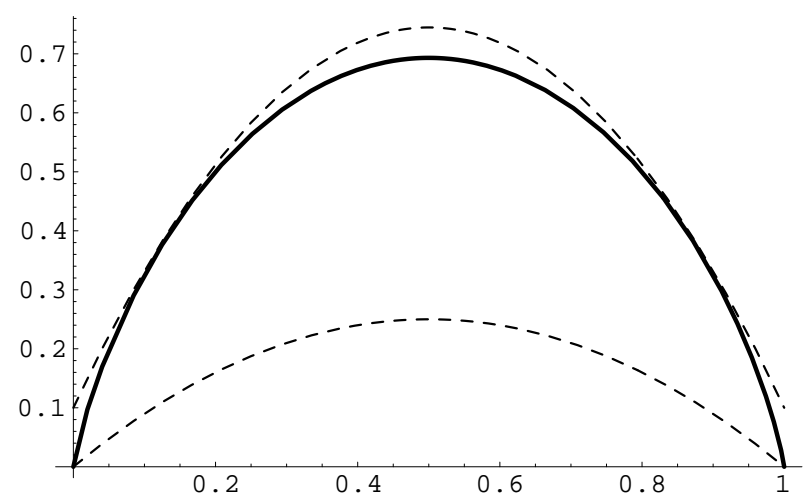

Figure 1: A quadratic upper and lower bound for the entropy function $\tilde{\eta}$.

\subsection{Quadratic approximation}

Equations (44) and (15) show the importance of the quantity $\operatorname{Tr} Q_{N}\left(\mathbb{1}-Q_{N}\right)$. It can be expressed in terms of the sequence $\{q(k)\}$ or, equivalently, of the Fourier transform $q^{\wedge}(\theta)=\chi_{K}(\theta)$ of the symbol $Q_{N}$. Using Equation (2),

$$
\begin{aligned}
\operatorname{Tr} & Q_{N}\left(\mathbb{1}-Q_{N}\right) \\
& =N q(0)-\sum_{n, m=1}^{N}|q(n-m)|^{2} \\
& =N q(0)-N \sum_{n=-(N-1)}^{N-1}\left(1-\frac{|n|}{N}\right)|q(n)|^{2} \\
& =N q(0)-N \int d \theta_{1} \int d \theta_{2} \chi_{K}\left(\theta_{1}\right) \chi_{K}\left(\theta_{2}\right) \sum_{n=-(N-1)}^{N-1}\left(1-\frac{|n|}{N}\right) \mathrm{e}^{i 2 \pi n\left(\theta_{1}-\theta_{2}\right)}
\end{aligned}
$$

Define the Dirichlet kernel,

$$
\begin{aligned}
k_{N}(\varphi) & :=\sum_{n=-(N-1)}^{N-1}\left(1-\frac{|n|}{N}\right) \mathrm{e}^{i 2 \pi n \varphi} \\
& =1+2 \sum_{n=1}^{N-1} \frac{N-n}{N} \cos 2 \pi n \varphi=\frac{1}{N} \frac{\sin ^{2} N \pi \varphi}{\sin ^{2} \pi \varphi}
\end{aligned}
$$

This is a sequence of positive normalised functions, weakly converging to the Dirac distribution,

$$
k_{N}(\varphi) \geq 0, \quad \int d \varphi k_{N}(\varphi)=1
$$


Therefore,

$$
\begin{aligned}
\operatorname{Tr} Q_{N}\left(\mathbb{1}-Q_{N}\right) & =N\left(\int d \theta \chi_{K}(\theta)-\int d \theta \int d \varphi \chi_{K}(\theta) \chi_{K}(\theta-\varphi) k_{N}(\varphi)\right) \\
& =N \int d \theta \int d \varphi \chi_{K}(\theta)\left[1-\chi_{K}(\theta-\varphi)\right] k_{N}(\varphi) \\
& =N \int d \varphi k_{N}(\varphi) \int d \theta \chi_{K}(\theta)\left[1-\chi_{K}(\theta-\varphi)\right] \\
& =N \int d \varphi k_{N}(\varphi)|K \backslash(K+\varphi)| .
\end{aligned}
$$

Note that both $S\left(Q_{N}\right)$ and $\operatorname{Tr} Q_{N}\left(\mathbb{1}-Q_{N}\right)$ are invariant for the replacement of $Q_{N}$ by $\mathbb{1}-Q_{N}$. As a consequence, Equation (6) can be written in the form

$$
\operatorname{Tr} Q_{N}\left(\mathbb{1}-Q_{N}\right)=N \int d \varphi k_{N}(\varphi)\left|K^{c} \backslash\left(K^{c}+\varphi\right)\right| .
$$

\section{$4 \quad$ Finitely many intervals}

As explained in Section 2 the subset $K$ of the torus $\mathbb{T}$ determines the state $\varphi$ we are studying. In this section we study sets $K$ composed of a finite number of intervals, whereas in the next section sets with an infinite number of intervals are treated.

\subsection{Lower bound}

By Equation (4) we have to bound $\operatorname{Tr} Q_{N}\left(\mathbb{1}-Q_{N}\right)$ from below. We consider a set

$K$ with a finite number of intervals, say $M$. Let $\delta>0$ be a fixed number which is smaller than any of the intervals and the holes between two such intervals. Therefore, $|K \backslash(K+\varphi)| \geq M \varphi$ for $0 \leq \varphi \leq \delta$. Equation (6) becomes,

$$
\begin{aligned}
S_{N} \geq & \operatorname{Tr} Q_{N}\left(\mathbb{1}-Q_{N}\right) \\
= & N \int d \varphi k_{N}(\varphi)|K \backslash(K+\varphi)| \\
\geq & 2 N M \int_{0}^{\delta} d \varphi k_{N}(\varphi) \varphi \\
= & 2 N M \int_{0}^{\delta} d \varphi \varphi\left[1+2 \sum_{n=1}^{N-1} \frac{N-n}{N} \cos 2 \pi n \varphi\right] \\
= & N M\left[\delta^{2}+\frac{2 \delta}{\pi} \sum_{n=1}^{N-1} \frac{\sin 2 \pi n \delta}{n}-\frac{2}{\pi^{2}} \sum_{n=1}^{N-1} \frac{\sin ^{2} \pi n \delta}{n^{2}}\right] \\
& +M\left[-\frac{2 \delta}{\pi} \sum_{n=1}^{N-1} \sin 2 \pi n \delta-\frac{1}{\pi^{2}} \sum_{n=1}^{N-1} \frac{\cos 2 \pi n \delta}{n}+\frac{1}{\pi^{2}} \sum_{n=1}^{N-1} \frac{1}{n}\right] .
\end{aligned}
$$


Using the identities,

$$
\sum_{n=1}^{+\infty} \frac{\sin 2 \pi n \delta}{n}=\frac{\pi}{2}(1-2 \delta) \quad \text { and } \quad \sum_{n=1}^{+\infty} \frac{\sin ^{2} \pi n \delta}{n^{2}}=\frac{\pi^{2}}{2} \delta(1-\delta)
$$

we obtain

$$
\begin{aligned}
S_{N} \geq N M\left[-\frac{2 \delta}{\pi} \sum_{n=N}^{+\infty} \frac{\sin 2 \pi n \delta}{n}+\frac{2}{\pi^{2}} \sum_{n=N}^{+\infty} \frac{\sin ^{2} \pi n \delta}{n^{2}}\right] \\
+M\left[-\frac{2 \delta}{\pi} \sum_{n=1}^{N-1} \sin 2 \pi n \delta-\frac{1}{\pi^{2}} \sum_{n=1}^{N-1} \frac{\cos 2 \pi n \delta}{n}+\frac{1}{\pi^{2}} \sum_{n=1}^{N-1} \frac{1}{n}\right] .
\end{aligned}
$$

Next, we estimate the different terms in (8) . The first term on the first line,

$$
\left|\sum_{n=N}^{+\infty} \frac{\sin 2 \pi n \delta}{n}\right|=\left|\sum_{n=N}^{+\infty} \frac{\cos \pi(2 n+1) \delta-\cos \pi(2 n-1) \delta}{2 n \sin \pi \delta}\right| \leq \frac{1}{N} \frac{1}{|\sin \pi \delta|}
$$

The second term on the first line,

$$
\left|\sum_{n=N}^{+\infty} \frac{\sin ^{2} \pi n \delta}{n^{2}}\right| \leq \sum_{n=N}^{+\infty} \frac{1}{n^{2}} \leq \int_{N-1}^{+\infty} d x \frac{1}{x^{2}}=\frac{1}{N-1}
$$

The first term on the second line,

$$
\left|\sum_{n=1}^{N-1} \sin 2 \pi n \delta\right| \leq \frac{1}{|\sin \pi \delta|}
$$

The second term on the second line, for any $\epsilon>0$ and $N$ sufficiently large,

$$
\left|\sum_{n=1}^{N-1} \frac{\cos 2 \pi n \delta}{n}\right| \leq-\log [2 \sin (2 \pi \delta)]+\epsilon .
$$

Finally, the last term on the last line,

$$
\sum_{n=1}^{N-1} \frac{1}{n} \geq \int_{1}^{N} d x \frac{1}{x}=\log N
$$

Putting everything together in (8), we find that there exists a constant $c_{1}>0$ independent of $N$ such that

$$
S_{N} \geq c_{1} \log N
$$




\subsection{Subadditivity}

Before establishing the upper bound for the entropy $S_{N}$ in case the set $K$ is composed of a finite number of intervals, we prove a general subadditivity property of this entropy. This will enable us to restrict the proof of the upper bound to the case of a single interval.

Suppose that $K_{1}$ and $K_{2}$ are disjoint sets and put $K:=K_{1} \cup K_{2}$. Denoting the symbols of these states by $Q, Q_{1}$ and $Q_{2}$, we shall prove the subadditivity property, namely,

$$
\operatorname{Tr} \tilde{\eta}\left(Q_{N}\right) \leq \operatorname{Tr} \tilde{\eta}\left(\left(Q_{1}\right)_{N}\right)+\operatorname{Tr} \tilde{\eta}\left(\left(Q_{2}\right)_{N}\right)
$$

To simplify notation, define $R:=Q_{N}, R_{1}:=\left(Q_{1}\right)_{N}$ and $R_{2}:=\left(Q_{2}\right)_{N}$. First, note that $R=R_{1}+R_{2}$. Remember that $\tilde{\eta}(x)=-x \log x-(1-x) \log (1-x)$ and thus $\tilde{\eta}^{\prime}(x)=-\log x+\log (1-x)$. We assume $R_{1}>0, R_{2}>0$ and $R_{1}+R_{2}<\mathbb{1}$. Otherwise, we can introduce operators $\tilde{R}_{1}:=(1-\epsilon) R_{1}+\frac{\epsilon}{2} \mathbb{1}$ and $\tilde{R}_{2}:=(1-\epsilon) R_{2}+\frac{\epsilon}{2} \mathbb{1}$, prove the subadditivity for these two operators and then take the limit $\epsilon \rightarrow 0$. Using the operator identity $\frac{d}{d \lambda} \operatorname{Tr} f(A+\lambda B)=\operatorname{Tr} B f^{\prime}(A+\lambda B)$,

$$
\begin{aligned}
\operatorname{Tr} \tilde{\eta}\left(R_{1}+R_{2}\right)-\operatorname{Tr} \tilde{\eta}\left(R_{1}\right) & =\left.\operatorname{Tr} \tilde{\eta}\left(R_{1}+\lambda R_{2}\right)\right|_{\lambda=0} ^{1} \\
& =\int_{0}^{1} d \lambda \frac{d}{d \lambda} \operatorname{Tr} \tilde{\eta}\left(R_{1}+\lambda R_{2}\right) \\
& =\int_{0}^{1} d \lambda \operatorname{Tr} R_{2} \log \frac{\mathbb{1}-R_{1}-\lambda R_{2}}{R_{1}+\lambda R_{2}}
\end{aligned}
$$

Because the inverse is operator decreasing,

$$
\frac{\mathbb{1}-R_{1}-\lambda R_{2}}{R_{1}+\lambda R_{2}}=\frac{1}{R_{1}+\lambda R_{2}}-\mathbb{1} \leq \frac{1}{\lambda R_{2}}-\mathbb{1}=\frac{\mathbb{1}-\lambda R_{2}}{\lambda R_{2}}
$$

and, because the logarithm is operator increasing,

$$
\log \frac{\mathbb{1}-R_{1}-\lambda R_{2}}{R_{1}+\lambda R_{2}} \leq \log \frac{\mathbb{1}-\lambda R_{2}}{\lambda R_{2}}
$$

Substituting this into Equation (11),

$$
\operatorname{Tr} \tilde{\eta}\left(R_{1}+R_{2}\right)-\operatorname{Tr} \tilde{\eta}\left(R_{1}\right) \leq \operatorname{Tr} \tilde{\eta}\left(R_{2}\right) .
$$

\subsection{Upper bound}

Due to subadditivity, it is enough to prove an upper bound for a set $K$ consisting of a single interval. We assume that the length of this interval $|K| \leq \frac{1}{2}$. Otherwise, we 
can work with $K^{c}$. By Equation (44) we have to bound $\operatorname{Tr} Q_{N}\left(\mathbb{1}-Q_{N}\right)$. In this case,

$$
|K \backslash(K+\varphi)|= \begin{cases}\varphi & \text { for }|\varphi| \leq|K| \\ |K| & \text { for }|K| \leq|\varphi| \leq \frac{1}{2}\end{cases}
$$

By Equation (6),

$$
\begin{aligned}
\operatorname{Tr} Q_{N}\left(\mathbb{1}-Q_{N}\right)= & 2 N \int_{0}^{\frac{1}{2}} d \varphi\left[1+2 \sum_{n=1}^{N-1} \frac{N-n}{N} \cos 2 \pi n \varphi\right]|K \backslash(K+\varphi)| \\
= & N\left[|K|(1-|K|)-\frac{2}{\pi^{2}} \sum_{n=1}^{N-1} \frac{\sin ^{2} \pi n|K|}{n^{2}}\right] \\
& +\frac{2}{\pi^{2}} \sum_{n=1}^{N-1} \frac{\sin ^{2} \pi n|K|}{n} .
\end{aligned}
$$

Using the identity,

$$
\sum_{n=1}^{+\infty} \frac{\sin ^{2} \pi n|K|}{n^{2}}=\frac{\pi^{2}}{2}|K|(1-|K|)
$$

we obtain,

$$
\operatorname{Tr} Q_{N}\left(\mathbb{1}-Q_{N}\right)=\frac{2 N}{\pi^{2}} \sum_{n=N}^{+\infty} \frac{\sin ^{2} \pi n|K|}{n^{2}}+\frac{2}{\pi^{2}} \sum_{n=1}^{N-1} \frac{\sin ^{2} \pi n|K|}{n} .
$$

The first term,

$$
\sum_{n=N}^{+\infty} \frac{\sin ^{2} \pi n|K|}{n^{2}} \leq \sum_{n=N}^{+\infty} \frac{1}{n^{2}} \leq \int_{N}^{+\infty} d x \frac{1}{x^{2}}=\frac{1}{N}
$$

The second term,

$$
\sum_{n=1}^{N-1} \frac{\sin ^{2} \pi n|K|}{n} \leq \sum_{n=1}^{N-1} \frac{1}{n} \leq 1+\int_{1}^{N-1} d x \frac{1}{x}=1+\log (N-1) .
$$

Putting everything together in (12), we find that there exists a constant $c_{2}$ independent of $N$ such that

$$
\operatorname{Tr} Q_{N}\left(\mathbb{1}-Q_{N}\right) \leq c_{2} \log N
$$

and, finally, by Equation (44), there exists a constant $c_{3}$ independent of $N$ such that

$$
S_{N} \leq c_{3}(\log N)^{2} .
$$

\subsection{Numerical results}

Analytically, we determined the asymptotics of the entropy $S_{N}$ between the $\log N$ lower bound (9) and the $(\log N)^{2}$ upper bound (13). In Figure 2 we present the results of a numerical calculation. The set $K$ consists of one interval of length $|K|=\frac{1}{2}$. The figure shows clearly the $\log N$ dependence. By the subadditivity property (10), we expect the same behaviour for all sets $K$ consisting of a finite number of intervals. 


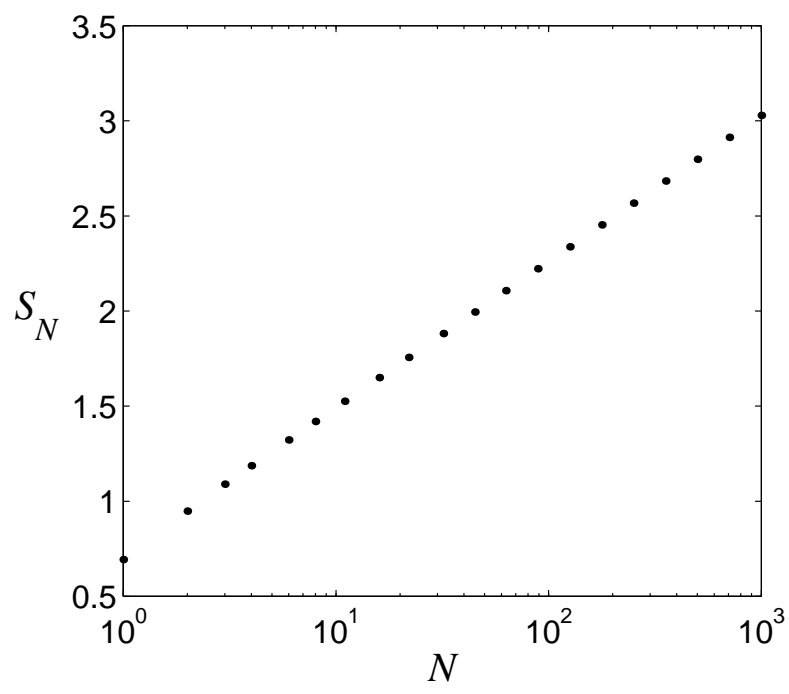

Figure 2: The entropy $S_{N}$ as a function of the length $N$ of the restriction, for an interval of length $\frac{1}{2}$ as set $K$. Notice the logarithmic scale.

\section{$5 \quad$ Infinitely many intervals}

For a set $K$ consisting of finitely many intervals, the entropy $S_{N}$ increases asymptotically slower than any power $N^{\alpha}$ with $\alpha>0$. However, it is easy to construct a state such that $S_{N}=N \log 2$. For example, one can take for $K$ a set of $2^{N-1}$ regularly spaced intervals, each of length $2^{-N}$. Note that this construction does not have an appropriate limit when $N \rightarrow \infty$. Nevertheless, it suggests that in the infinitely many intervals case the entropy $S_{N}$ could have a richer behaviour. This will be shown in the present section by presenting a class of states for which the growth exponent $\alpha$ can take any value $\alpha \in(0,1)$.

\subsection{A Cantor-like construction}

The standard Cantor set is constructed recursively by removing in step $m$ a fixed fraction of the set obtained in step $m-1$. This would leave us with a set of zero Lebesgue measure. To avoid this, we remove a fraction in step $m$ which decreases with $m$, such that the limit set has strictly positive Lebesgue measure.

We start with the unit interval. Remove in the first step an open interval in the middle of the unit interval with length $1-\gamma(1)$. The resulting set $K_{1}$ consists of two closed intervals each of length $\gamma(1) / 2$. In the second step, for each of these two intervals, a fraction $1-\gamma(2)$ is removed in the middle of these intervals. This leaves us with a set $K_{2}$ consisting of four closed intervals of length $\gamma(1) \gamma(2) / 4$. Continuing this procedure, 
in step $m$ we obtain a set $K_{m}$ of $2^{m}$ closed intervals of length

$$
\ell_{i}(m):=\frac{\prod_{n=1}^{m} \gamma(n)}{2^{m}}
$$

There are $2^{m}-1$ holes in between such intervals, $2^{m-1}$ of which are created in step $m$. The latter have a length

$$
\ell_{h}(m):=\ell_{i}(m-1)(1-\gamma(m))=\frac{\prod_{n=1}^{m-1} \gamma(n)}{2^{m-1}}(1-\gamma(m))
$$

The Lebesgue measure of the limit set $K$ is then $\prod_{n=1}^{\infty} \gamma(n)$.

To construct an explicit example, we have to fix a function $m \mapsto \gamma(m)$. We can as well specify the function $m \mapsto \ell_{h}(m)=a q^{m}$, where $0<q<\frac{1}{2}$ and $a$ is chosen such that

$$
1>\sum_{m=1}^{+\infty} 2^{m-1} a q^{m}=\frac{a}{2} \frac{2 q}{1-2 q} .
$$

The resulting set has Lebesgue measure $1-a q /(1-2 q)$.

\subsection{Lower bound}

To bound $\operatorname{Tr} Q_{N}\left(\mathbb{1}-Q_{N}\right)$ from below, we start from Equation (6) $)$. As before, $K_{m}$ denotes the set obtained after $m$ steps in the construction of the Cantor-like set $K$. Then $K_{m}$ is the union of $2^{m}$ intervals, each of length $l_{i}(m)$. Because $K \subset K_{m}$, (6) can be estimated by

$$
\begin{aligned}
\operatorname{Tr} Q_{N}\left(\mathbb{1}-Q_{N}\right) & =N \int_{-\frac{1}{2}}^{\frac{1}{2}} d \varphi k_{N}(\varphi)|K \backslash(K+\varphi)| \\
& \geq N \int_{-\frac{1}{2}}^{\frac{1}{2}} d \varphi k_{N}(\varphi)\left|K \backslash\left(K_{m}+\varphi\right)\right|
\end{aligned}
$$

and since $k_{N}(\varphi) \geq N / \pi^{2}$ when $|\varphi| \leq 1 / 2 N$,

$$
\begin{aligned}
\operatorname{Tr} Q_{N}\left(\mathbb{1}-Q_{N}\right) & \geq \frac{1}{\pi^{2}} N^{2} \int_{-\frac{1}{2 N}}^{\frac{1}{2 N}} d \varphi\left|K \backslash\left(K_{m}+\varphi\right)\right| \\
& =\frac{2}{\pi^{2}} N^{2} \int_{0}^{\frac{1}{2 N}} d \varphi\left|K \backslash\left(K_{m}+\varphi\right)\right|
\end{aligned}
$$

For given $N$, take $m$ such that

$$
\ell_{h}(m) \geq \frac{1}{2 N}>\ell_{h}(m+1)
$$


As $K_{m}$ consists of $2^{m}$ translations of the interval $\left[0, \ell_{i}(m)\right]$, the Cantor-like set $K$ consists of $2^{m}$ translations of another Cantor-like set $\tilde{K} \subset\left[0, \ell_{i}(m)\right]$. Let us denote these translations by $x_{\ell}+\left[0, \ell_{i}(m)\right]$ and $x_{\ell}+\tilde{K}$ for $\ell=1, \ldots, 2^{m}$.

For $\varphi \in[0,1 / 2 N], \varphi \leq \ell_{h}(k)$ for all $k=1, \ldots, m$. This means that a translation by $\varphi$ of an interval of length $\ell_{i}(m)$ in $K_{m}$ will never bridge the hole (of length $\ell_{h}(k), k=$ $1, \ldots, m)$ between this interval and the next. Therefore, every $x_{\ell}+\tilde{K}$ will overlap with one and only one $x_{\tilde{\ell}}+\left[0, \ell_{i}(m)\right]+\varphi$, namely the one with $\tilde{\ell}=\ell$. As a consequence,

$$
\left|K \backslash\left(K_{m}+\varphi\right)\right|=2^{m}\left|\tilde{K} \backslash\left(\left[0, \ell_{i}(m)\right]+\varphi\right)\right|=2^{m}\left|\tilde{K} \backslash\left[\varphi, \ell_{i}(m)\right]\right|=2^{m}|\tilde{K} \cap[0, \varphi]| .
$$

This quantity has to be estimated from below. For $\varphi \in(0,1 / 2 N]$, take $n$ such that $\ell_{i}(n) \geq \varphi>\ell_{i}(n+1)$. Then,

$$
|\tilde{K} \cap[0, \varphi]| \geq \ell_{i}(n+1) \prod_{k=n+2}^{+\infty} \gamma(k)=\frac{1}{2^{n+1}} \prod_{k=1}^{+\infty} \gamma(k),
$$

and

$$
\varphi \leq \ell_{i}(n)=\frac{1}{2^{n}} \prod_{k=1}^{n} \gamma(k)
$$

which gives

$$
\frac{|\tilde{K} \cap[0, \varphi]|}{\varphi} \geq \frac{1}{2} \prod_{k=n+1}^{+\infty} \gamma(k) \geq \frac{1}{2}|K|
$$

and so

$$
|\tilde{K} \cap[0, \varphi]| \geq \frac{1}{2}|K| \varphi
$$

which does not depend any longer on $n$. It follows that

$$
\operatorname{Tr} Q_{N}\left(\mathbb{1}-Q_{N}\right) \geq 2^{m} \frac{1}{\pi^{2}}|K| N^{2} \int_{0}^{\frac{1}{2 N}} d \varphi \varphi=2^{m} \frac{1}{8 \pi^{2}}|K| .
$$

This is an estimate from below of $\operatorname{Tr} Q_{N}\left(\mathbb{1}-Q_{N}\right)$ where $N$ and $m$ are coupled by (16). From the latter we also have that $N<1 / 2 \ell_{h}(m+1)$. Therefore,

$$
\frac{\log \operatorname{Tr} Q_{N}\left(I-Q_{N}\right)}{\log N}>\frac{\log \left(2^{m} \frac{1}{8 \pi^{2}}|K|\right)}{-\log \left(2 \ell_{h}(m+1)\right)} .
$$

The limit $N \rightarrow \infty$ corresponds to the limit $m \rightarrow \infty$. Using the explicit form $\ell_{h}(m)=$ $a q^{m}$, we finally get

$$
\liminf _{N \rightarrow+\infty} \frac{\log \operatorname{Tr} Q_{N}\left(I-Q_{N}\right)}{\log N} \geq \frac{\log 2}{-\log q}
$$




\subsection{Upper bound}

To get an upper bound for $\operatorname{Tr} Q_{N}\left(\mathbb{1}-Q_{N}\right)$, we start from Equation (17). With $C(\varphi):=$ $\left|K^{c} \backslash\left(K^{c}+\varphi\right)\right|$, it reads

$$
\operatorname{Tr} Q_{N}\left(\mathbb{1}-Q_{N}\right)=N \int d \varphi k_{N}(\varphi) C(\varphi),
$$

For $\theta>0$, take $m$ such that

$$
\ell_{h}(m) \geq \theta>\ell_{h}(m+1) .
$$

We bound $C(\theta)$ from above,

$$
\begin{aligned}
C(\theta) & \leq \sum_{k=1}^{\infty} 2^{k-1} \min \left\{\theta, \ell_{h}(k)\right\} \\
& =\sum_{k=1}^{m} 2^{k-1} \theta+\sum_{k=m+1}^{\infty} 2^{k-1} \ell_{h}(k) \\
& \leq\left(2^{m}-1\right) \ell_{h}(m)+\sum_{k=m+1}^{\infty} 2^{k-1} \ell_{h}(k) \\
& \leq 2 \prod_{n=1}^{m-1} \gamma(n)(1-\gamma(m))+\sum_{k=m+1}^{\infty} \prod_{n=1}^{k-1} \gamma(n)(1-\gamma(k)) .
\end{aligned}
$$

Obviously, this bound increases with $\theta$. The kernel $k_{N}(\varphi)$ satisfies

$$
k_{N}(\varphi) \leq \begin{cases}N & \text { for }|\varphi| \leq \theta \\ \frac{\pi^{2}}{2 N} \frac{1}{\varphi^{2}} & \text { for }|\varphi| \geq \theta\end{cases}
$$

and so we find

$$
\begin{aligned}
\operatorname{Tr} Q_{N}\left(\mathbb{1}-Q_{N}\right) & \leq N^{2} \int_{|\varphi| \leq \theta} d \varphi C(\varphi)+\int_{|\varphi| \geq \theta} d \varphi C(\varphi) \frac{\pi^{2}}{2} \frac{1}{\varphi^{2}} \\
& \leq 2 N^{2} \theta C(\theta)+\frac{\pi^{2}}{2} \sum_{k=0}^{m}\left(\ell_{h}(k)-\ell_{h}(k+1)\right) \frac{C\left(\ell_{h}(k)\right)}{\ell_{h}(k+1)^{2}} \\
& \leq 2 N^{2} \ell_{h}(m) C(\theta)+\frac{\pi^{2}}{2} \sum_{k=0}^{m} \frac{\ell_{h}(k) C\left(\ell_{h}(k)\right)}{\ell_{h}(k+1)^{2}} .
\end{aligned}
$$

Take again the explicit form $\ell_{h}(m)=a q^{m}$. Then

$$
\prod_{n=1}^{m-1} \gamma(n)(1-\gamma(m))=2^{m-1} \ell_{h}(m)=\frac{a}{2}(2 q)^{m},
$$


and (19) becomes

$$
\begin{aligned}
C(\theta) & \leq 2 \prod_{n=1}^{m-1} \gamma(n)(1-\gamma(m))+\sum_{k=m+1}^{\infty} \prod_{n=1}^{k-1} \gamma(n)(1-\gamma(k)) \\
& =2 \frac{a}{2}(2 q)^{m}+\sum_{k=m+1}^{\infty} \frac{a}{2}(2 q)^{k}=\frac{a(1-q)}{1-2 q}(2 q)^{m} .
\end{aligned}
$$

If $\theta=\ell_{h}(k)$, then by (18), we have to put $m=k$, and so

$$
C\left(\ell_{h}(k)\right) \leq \frac{a(1-q)}{1-2 q}(2 q)^{k}
$$

Substituting inequalities (21) and (22) into (20), we find

$$
\begin{aligned}
\operatorname{Tr} Q_{N}\left(\mathbb{1}-Q_{N}\right) & \leq 2 N^{2} a q^{m} \frac{a(1-q)}{1-2 q}(2 q)^{m}+\frac{\pi^{2}}{2} \sum_{k=0}^{m} \frac{a q^{k}}{\left(a q^{k+1}\right)^{2}} \frac{a(1-q)}{1-2 q}(2 q)^{k} \\
& =2 N^{2} a q^{m} \frac{a(1-q)}{1-2 q}(2 q)^{m}+\frac{\pi^{2}}{2} \frac{1}{q^{2}} \frac{2(1-q)}{1-2 q}\left(2^{m+1}-1\right) \\
& \leq \frac{2(1-q)}{1-2 q}\left[N^{2} a^{2}\left(2 q^{2}\right)^{m}+\frac{\pi^{2}}{2} \frac{1}{q^{2}} 2^{m}\right] \\
& =: c_{1} N^{2}\left(2 q^{2}\right)^{m}+c_{2} 2^{m},
\end{aligned}
$$

where $c_{1}$ and $c_{2}$ are independent of $N$.

To get an upper bound as a function of $N$, we have to fix a function $m(N)$ and plug it into (23). Let

$$
\gamma:=\frac{\log 2}{-\log q}=\frac{1}{-\log _{2} q}
$$

then choose $m$ to be

$$
m=\left[\log _{2 q^{2}} N^{\gamma-2}\right] \leq \log _{2 q^{2}} N^{\gamma-2}=\frac{\log _{2} N^{\gamma-2}}{\log _{2} 2 q^{2}},
$$

where $[a]$ denotes the integer part of the number $a$. Then

$$
N^{2}\left(2 q^{2}\right)^{m} \leq N^{\gamma}
$$

and

$$
2^{m} \leq\left(2^{\log _{2} N^{\gamma-2}}\right)^{\frac{1}{\log _{2} 2 q^{2}}}=N^{\frac{\gamma-2}{1+\log _{2} q^{2}}}=N^{\gamma}
$$

and so

$$
\operatorname{Tr} Q_{N}\left(\mathbb{1}-Q_{N}\right) \leq\left(c_{1}+c_{2}\right) N^{\gamma}
$$

from which we get the upper bound

$$
\limsup _{N \rightarrow+\infty} \frac{\log \operatorname{Tr} Q_{N}\left(\mathbb{1}-Q_{N}\right)}{\log N} \leq \gamma=\frac{\log 2}{-\log q} .
$$


Combining the results (17) and (24) we see that $\lim _{N \rightarrow+\infty} \log \operatorname{Tr} Q_{N}\left(\mathbb{1}-Q_{N}\right) / \log N$ exists, which implies that also $\lim _{N \rightarrow+\infty} \log S_{N} / \log N$ exists, and

$$
\alpha=\lim _{N \rightarrow+\infty} \frac{\log S_{N}}{\log N}=\lim _{N \rightarrow+\infty} \frac{\log \operatorname{Tr} Q_{N}\left(\mathbb{1}-Q_{N}\right)}{\log N}=\frac{\log 2}{-\log q} .
$$

Since $q$ can be any number in the interval $(0,1 / 2)$, the growth exponent $\alpha$ can take any value in $(0,1)$.

\section{References}

[1] R. Alicki, M. Fannes, Quantum Dynamical Systems, Oxford University Press, Oxford, 2001.

[2] O. Bratteli, D.W. Robinson, Operator Algebras and Quantum Statistical Mechanics, Vol. 2, Springer-Verlag, New York, 1997.

[3] D.E. Evans, Y. Kawahigashi, Quantum Symmetries on Operator Algebras, Oxford University Press, Oxford, 1998.

[4] M. Fannes, B. Nachtergaele, R. F. Werner, Finitely correlated states on quantum spin chains, Comm. Math. Phys. 144, 443-490 (1992).

[5] U. Grenander, G. Szegö, Toeplitz Forms and their Applications, University of California Press, 1958.

[6] F. Hiai, D. Petz, The proper formula for relative entropy and its asymptotics in quantum probability, Comm. Math. Phys. 143, 99-114 (1991).

[7] M.A. Nielsen, I.L. Chuang, Quantum Computation and Quantum Information, Cambridge University Press, Cambridge, 2000.

[8] I. Peschel, X. Wang, M. Kaulke, K. Hallberg (Eds.), Density Matrix Renormalization, LNP 528, Springer, Berlin, Heidelberg, 1999. 